\title{
NONLINEAR DARK SOLITARY SH WAVES IN A HETEROGENEOUS LAYER
}

\author{
D. DEMIRKUŞ ${ }^{1}, \S$
}

\begin{abstract}
In this study, we consider the nonlinear propagation of shear horizontal $(\mathrm{SH})$ waves in a layer of finite thickness. The materials of the layer are assumed to be heterogeneous, isotropic, and generalized neo-Hookean. We assume that heterogeneity varies only with the thickness and we choose hyperbolic functions for heterogeneity type. We also assume that the traction is free on the upper surface of the layer. Furthermore, the lower boundary is rigidly fixed. Using a perturbation method and keeping the balance of the nonlinearity and the dispersion in the analysis, we show that the self-modulation of nonlinear SH waves can be given by the nonlinear Schrödinger (NLS) equation. Using well known solutions of NLS equation, we find that the dark solitary SH waves can exist depending on the nonlinear constitution of the layer. Consequently, the effects of the heterogeneity and the nonlinearity on the deformation field are considered for these waves.
\end{abstract}

Keywords: Nonlinear SH waves, dark solitary waves, rigid substratum, heterogeneity.

AMS Subject Classification: 35-XX, 35B20, 35Q55.

\section{INTRODUCTION}

In seismology we see some examples of the seismic waves such as body waves, surface waves, Love waves, and Rayleigh waves. In this study, we consider shear waves which are one type of body waves. If shear waves are polarized horizontally, then such waves are called shear horizontal (SH) waves. In general, the seismic waves are not dispersive in an unbounded homogeneous medium. Using the wave guides it is possible to obtain dispersive waves. Seismology, geophysics, and electronic signal processing devices are some examples of application areas for dispersive elastic waves. More information about applications and for reviews, we refer to [1] and references therein.

This work includes nonlinear elastic waves in a heterogeneous medium, so we need to overview some nonlinear works such as from [1] to [10]. In these works, the effect of constitutional nonlinearity on the propagation characteristics of dispersive elastic waves are studied in a homogeneous medium. Furthermore, for some linear works in a heterogeneous medium we refer to from [11] to [13]. In [14], we investigate nonlinear bright solitary

\footnotetext{
${ }^{1}$ Beykent University- Faculty of Science and Letters - Department of Mathematics - Istanbul - Turkey. e-mail: dilekdemirkus@beykent.edu.tr; ORCID: https://orcid.org/0000-0002-6544-3668.

$\S$ Manuscript received: June 26, 2019; accepted: September 10, 2019.

TWMS Journal of Applied and Engineering Mathematics, Vol.11, No.2 (C) Işılk University, Department of Mathematics, 2021; all rights reserved.
} 
SH waves in a hyperbolically heterogeneous layer; whereas, in this paper we investigate nonlinear dark solitary SH waves in a hyperbolically heterogeneous layer. Moreover, In $[15,16,17]$, we consider nonlinear SH waves in a plate. Plate case may be considered a little bit an improved version of a layer in terms of geometry, i.e. a layer may be though as a half of a plate. Due to different boundary conditions, we need to consider as different problems.

The present work searches for the propagation of nonlinear SH waves in a heterogeneous, isotropic, and generalized neo-Hookean layer overlying a rigid substratum. We assume that heterogeneity varies with the thickness and we choose hyperbolic function of one variable as a heterogeneity type. Moreover, the traction is free on the upper surface of the layer and the lower boundary is rigidly fixed. Applying the method of multiple scales and striking a balance between the weak nonlinearity and the dispersion in the asymptotic analysis, we derive a nonlinear Schrödinger (NLS) equation describing a self-modulation of nonlinear $\mathrm{SH}$ waves. We claim that the existence of dark solitary $\mathrm{SH}$ waves depends on the nonlinear constitution of the layer, and consider the effects of the heterogeneity and the nonlinearity on the propagation characteristics of SH waves via NLS equation. Furthermore, the effects of the heterogeneity and the nonlinearity on the deformation field are considered for these waves.

\section{Formulation of the Problem}

The work [14] can be seen for the anti-plane motion of the considered problem. Our aim is to overcome with the propagation of nonlinear SH waves, so it is necessary to define $\mathrm{SH}$ wave such that

$$
x=X, y=Y, z=Z+u(X, Y, t) .
$$

Here, $(x, y, z)$ and $(X, Y, Z)$ are the spatial and material coordinates respectively, $u=$ $u(X, Y, t)$ is the displacement in the $Z$-direction, and $t$ is the time. Furthermore, the thickness of this layer is $h, X$-axis is for the propagation of the waves, and $Z$-axis is for the motion of the particle. The layer is in the region between the planes $Y=0$ and $Y=h$, and also the semi-infinite substratum occupies the region $Y<0$. The displacement in the $Z$-direction is zero at the rigid boundary $Y=0$ and the boundary $Y=h$ is assumed to be free of traction. Moreover, we consider heterogeneous, isotropic, and generalized neo-Hookean materials in such a layer. Under some restriction for more detail see [14], the governing equation and the boundary conditions can be written as

$$
\begin{aligned}
& \frac{\partial^{2} u}{\partial t^{2}}-c_{T}^{2}\left(\frac{\partial^{2} u}{\partial X^{2}}+\frac{\partial^{2} u}{\partial Y^{2}}\right)-\frac{1}{\rho} \frac{\partial\left(\rho c_{T}^{2}\right)}{\partial Y} \frac{\partial u}{\partial Y}= \\
& n_{T}\left[\frac{\partial}{\partial X}\left(\frac{\partial u}{\partial X} \mathcal{K}(u)\right)+\frac{\partial}{\partial Y}\left(\frac{\partial u}{\partial Y} \mathcal{K}(u)\right)\right]+\frac{\mathcal{K}(u)}{\rho} \frac{\partial\left(\rho n_{T}\right)}{\partial Y} \frac{\partial u}{\partial Y}, \\
& \frac{\partial u}{\partial Y}+\frac{n_{T}}{c_{T}^{2}} \mathcal{K}(u) \frac{\partial u}{\partial Y}=0 \quad \text { on } Y=h \quad \text { and } \quad u=0 \quad \text { on } Y=0 .
\end{aligned}
$$

Here, the linear shear wave velocity and the nonlinear material function can be denoted by $c_{T}=\sqrt{\mu / \rho}$ and $n_{T}$ respectively, and $\mathcal{K}$ can be defined by

$$
\mathcal{K}(u)=(\partial u / \partial X)^{2}+(\partial u / \partial Y)^{2} .
$$

Because of the homogeneity, we note that the functions $\mu, \rho$ and $n_{T}$ are constants in [10], but here they are not. The constituent material of the layer softens in shear if $n_{T}<0$, but if $n_{T}>0$ it hardens. In this study, $n_{T}$ indicates a continuously differentiable function 
of $Y$, and also because of heterogeneity we need to put the following conditions on the functions $\mu$ and $\rho$

$$
\mu=\mu_{0} \cosh ^{2}(\alpha Y), \quad \rho=\rho_{0} \cosh ^{2}(\alpha Y) .
$$

Here, $\mu_{0}$ and $\rho_{0}$ are constants, and $\alpha$ is a parameter. We choose condition (5) for simplicity. In this paper, we consider not only heterogeneity but also nonlinearity. Therefore, it is not easy to overcome with two difficulties.

\section{Nonlinear Self-Modulation of SH Waves}

The method of multiple scales [18] is a good method for solving such a nonlinear problem. For applying the method, it is necessary to introduce new variables as

$$
x_{i}=\varepsilon^{i} X, \quad t_{i}=\varepsilon^{i} t, \quad y=Y, \quad i=0,1,2 .
$$

Here, $x_{1}, x_{2}, t_{1}, t_{2}$ indicate the slow variables, $x_{0}, y, t_{0}$ indicate the fast variables, and $\varepsilon$ indicates a small positive parameter. Then, $u$ can be considered as a function of (6), and $u$ can be expanded in the following asymptotic series in $\varepsilon$;

$$
u=\sum_{n=1}^{\infty} \varepsilon^{n} u_{n}\left(x_{0}, x_{1}, x_{2}, y, t_{0}, t_{1}, t_{2}\right) .
$$

If we plug this asymptotic series with (6) into equation (2) and the boundary conditions (3), then we can get a system of equations and boundary conditions for determining $u_{n}$ successively. Up to the third-order in $\varepsilon$ they can be written as follows:

$$
\begin{aligned}
& \mathcal{O}(\varepsilon): \quad \mathcal{L}_{0} u_{1}=0 \\
& \frac{\partial u_{1}}{\partial y}=0 \quad \text { on } \quad y=h \quad \text { and } \quad u_{1}=0 \quad \text { on } \quad y=0 \text {; } \\
& \mathcal{O}\left(\varepsilon^{2}\right): \quad \mathcal{L}_{0} u_{2}=\mathcal{L}_{1} u_{1}, \\
& \frac{\partial u_{2}}{\partial y}=0 \quad \text { on } \quad y=h \quad \text { and } \quad u_{2}=0 \quad \text { on } \quad y=0 \text {; } \\
& \mathcal{O}\left(\varepsilon^{3}\right): \quad \mathcal{L}_{0} u_{3}=\mathcal{L}_{1} u_{2}+\mathcal{L}_{2} u_{1}+\mathcal{N} u_{1}, \\
& \frac{\partial u_{3}}{\partial y}=0 \quad \text { on } \quad y=h \quad \text { and } \quad u_{3}=0 \quad \text { on } \quad y=0 \text {; }
\end{aligned}
$$

The differential operators $\mathcal{L}_{0}, \mathcal{L}_{1}, \mathcal{L}_{2}, \mathcal{K}$, and $\mathcal{N}$ can be defined by

$$
\begin{aligned}
\mathcal{L}_{0} & =\frac{\partial^{2}}{\partial t_{0}^{2}}-c_{T}^{2}\left(\frac{\partial^{2}}{\partial x_{0}^{2}}+\frac{\partial^{2}}{\partial y^{2}}\right)-\frac{\mu^{\prime}}{\rho} \frac{\partial}{\partial y}, \\
\mathcal{L}_{1} & =2\left(c_{T}^{2} \frac{\partial^{2}}{\partial x_{0} \partial x_{1}}-\frac{\partial^{2}}{\partial t_{0} \partial t_{1}}\right), \\
\mathcal{L}_{2} & =c_{T}^{2}\left(\frac{\partial^{2}}{\partial x_{1}^{2}}+2 \frac{\partial^{2}}{\partial x_{0} \partial x_{2}}\right)-\frac{\partial^{2}}{\partial t_{1}^{2}}-2 \frac{\partial^{2}}{\partial t_{0} \partial t_{2}}, \\
\mathcal{K} & =\left(\frac{\partial}{\partial x_{0}}\right)^{2}+\left(\frac{\partial}{\partial y}\right)^{2}, \\
\mathcal{N} & =\frac{1}{\rho}\left[\frac{\partial}{\partial x_{0}}\left(\rho n_{T} \mathcal{K} \frac{\partial}{\partial x_{0}}\right)+\frac{\partial}{\partial y}\left(\rho n_{T} \mathcal{K} \frac{\partial}{\partial y}\right)\right] .
\end{aligned}
$$

We emphasize that it is possible to reduce the obtained order problems to the order problems [10]. We also emphasize that the obtained order problems are linear at each step 
and the solution of the linear case of this problem is identical with the solution of the firstorder problem. Furthermore, we observe that the main analysis is same as the analysis in [14], but the results are different. So, we consider only the first-order perturbation problem here, let

$$
u_{1}=\mu_{0}^{-\frac{1}{2}} \operatorname{sech}(\alpha y) \sum_{l=1}^{\infty}\left[A_{1}^{(l)} e^{i l k s_{l} y}+B_{1}^{(l)} e^{-i l k s_{l} y}\right] e^{i l \phi}+\text { c.c. }
$$

be for the solution of equation (8) where

$$
s_{l}=\left[\left(c^{2} / c_{0_{T}}^{2}\right)-\left(\alpha^{2} /\left(k^{2} l^{2}\right)\right)-1\right]^{1 / 2}, \quad \phi=k x_{0}-\omega t_{0}, \quad c_{0_{T}}=\sqrt{\mu_{0} / \rho_{0}},
$$

$k, \omega$, and $c=\omega / k$ indicate the wave number, the angular frequency, and the phase velocity respectively. Moreover, $l$ indicates a positive integer, c.c. indicates the complex conjugate to the preceding terms, and also $A_{1}^{(l)}$ and $B_{1}^{(l)}$ indicate the first-order amplitude functions of wave propagation depending on the slow variables $x_{1}, x_{2}, t_{1}$, and $t_{2}$. The case $c>c_{0_{T}}\left[1+\left(\alpha^{2} /\left(k^{2} l^{2}\right)\right)\right]^{1 / 2}$ is for the validity of the analysis; otherwise, the propagation of SH waves is not possible in this layer. Plugging the form of the solution (15) into the boundary conditions (9) yields

$$
\mathbf{W}_{l} \mathbf{U}_{1}^{(l)}=\mathbf{0}
$$

where $\mathbf{W}_{l}$ denotes the dispersion matrix

$$
\mathbf{W}_{l}=\left[\begin{array}{cc}
\left(-\alpha \tanh (\alpha h)+i l k s_{l}\right) e^{i l k s_{l} h} & \left(-\alpha \tanh (\alpha h)-i l k s_{l}\right) e^{-i l k s_{l} h} \\
1 & 1
\end{array}\right]
$$

and

$$
\mathbf{U}_{1}^{(l)}=\left[A_{1}^{(l)}, B_{1}^{(l)}\right]^{T} .
$$

For this paper the nonlinear self-modulation is enough to talk, and the harmonicresonance phenomena is not included, so the following condition may be assumed

$$
\operatorname{det} \mathbf{W}_{l} \neq 0 \quad \text { for } \quad l \neq 1 \text {. }
$$

To obtain the nontrivial solution of system (17) for $l=1$, the condition $\operatorname{det} \mathbf{W}_{1}=0$ is a must step. Then, using the condition $\operatorname{det} \mathbf{W}_{1}=0$ we can find the dispersion relation as follows:

$$
k p \cos (k p h)-\alpha \tanh (\alpha h) \sin (k p h)=0
$$

where $p=s_{1}$. Therefore,

$$
k p h=\arctan [k p /(\alpha \tanh (\alpha h))]+n \pi .
$$

Here, the branches of the dispersion relation can be denoted by $n(n=0,1,2, \ldots)$. We observe that dispersion relation (22) as $\alpha \rightarrow 0$ is the same as the dispersion relation of [10]. Under the consideration above, we give the solution of system (17) as

$$
\begin{aligned}
& \mathbf{U}_{1}^{(l)}=\mathcal{A}_{1} \mathbf{R} \text { for } l=1 \text { and } \\
& \mathbf{U}_{1}^{(l)}=\mathbf{0} \text { for } l \neq 1
\end{aligned}
$$

where $\mathcal{A}_{1}$ is a complex function of the slow variables and $\mathbf{R}$ is a vector satisfying

$$
\mathbf{W}_{1} \mathbf{R}=\mathbf{0} .
$$

Using (24), we get the vector $\mathbf{R}$, as

$$
\mathbf{R}=\left[\begin{array}{ll}
R_{1}, & R_{2}
\end{array}\right]^{T}=\left[1, \frac{-\alpha \tanh (\alpha h)+i k p}{\alpha \tanh (\alpha h)+i k p} e^{2 i k p h}\right]^{T} .
$$


Then, the first-order solution can be given by

$$
u_{1}=\mu_{0}^{-\frac{1}{2}} \mathcal{A}_{1} \operatorname{sech}(\alpha y)\left(R_{1} e^{i k p y}+R_{2} e^{-i k p y}\right) e^{i \phi}+\text { c.c.. }
$$

For solution (26), it is necessary to find the function $\mathcal{A}_{1}$, explicitly. Using the compatibility condition of the second-order problem we can find the function $\mathcal{A}_{1}$ such that $\mathcal{A}_{1}=\mathcal{A}_{1}\left(x_{1}-V_{g} t_{1}, x_{2}, t_{2}\right)$, but it is not clear exactly. Moreover, using the third-order problem and defining the following nondimensional variables and constants

$$
\tau=\omega t_{2}, \quad \xi=k\left(x_{1}-V_{g} t_{1}\right), \quad \mathcal{A}=k \mathcal{A}_{1}, \quad \Gamma=k^{2} \tilde{\Gamma} / \omega, \quad \Delta=\tilde{\Delta} / \omega k^{2}
$$

then we get nonlinear Schrödinger (NLS) equation as follows:

$$
i \frac{\partial \mathcal{A}}{\partial \tau}+\Gamma \frac{\partial^{2} \mathcal{A}}{\partial \xi^{2}}+\Delta|\mathcal{A}|^{2} \mathcal{A}=0
$$

where

$$
\tilde{\Gamma}=\frac{1}{2} \frac{d^{2} \omega}{d k^{2}} \quad \text { and } \quad \tilde{\Delta}=-\frac{\mathbf{L} \cdot \mathbf{F}}{\mathbf{L} \frac{\partial \mathbf{W}_{1}}{\partial \omega} \mathbf{R}} .
$$

Here, we define the vectors $\mathbf{L}$ and $\mathbf{F}$ in [14] and the analysis is same as the work [14], so for more detail we refer to [14]. Using known properties of solutions of the NLS equation in the following section, we can discuss the existence of dark solitary SH waves in such a layer.

\section{Conclusion}

In wave propagation, the NLS equation is a famous characteristic equation. We know that the sign of $\Gamma \Delta$ behaves as a criteria for determining how a given initial data will evolve for long times for the asymptotic wave field governed by the NLS equation. For $\Gamma \Delta<0$, a solution of the form

$$
\mathcal{A}(\xi, \tau)=\phi(\eta) e^{i\left[\Gamma^{2} \Delta \phi_{0}^{2} \tau-F(\eta)\right]}, \quad \eta=\xi-V_{0} \tau
$$

which tends to the uniform solution $\phi_{0} e^{i \Gamma^{2} \Delta \phi_{0}^{2} \tau}$ as $|\eta| \rightarrow \infty$ exists. The solutions for $\phi$ and $F$ are found to be

$$
\phi^{2}=\phi_{0}^{2}\left(1-\sin ^{2} B_{0} \operatorname{sech}^{2} \psi\right) \quad \text { and } \quad F=\arctan \left(\tan B_{0} \tanh \psi\right)
$$

where $B_{0}$ is a constant, $\psi$ and $V_{0}$ are given as

$$
\psi=(-\Gamma \Delta / 2)^{1 / 2} \phi_{0} \eta \sin B_{0} \quad \text { and } \quad V_{0}= \pm 2^{-3 / 2} \Gamma(-\Gamma \Delta)^{1 / 2} \phi_{0} .
$$

Solution (30) is called as a dark solitary wave solution, for more details see [19, 20, 21]. In this study, the following limits are valid for all branches of dispersion relation (22)

$$
C \rightarrow \infty, V_{G} \rightarrow 0 \text { as } K \rightarrow 0 \text { and } C \rightarrow 1, V_{G} \rightarrow 1 \text { as } K \rightarrow \infty
$$

where $C=c / c_{0_{T}}, V_{G}=V_{g} / c_{0_{T}}$, and $K=k h$ denote the nondimensional phase velocity, the nondimensional group velocity, and the nondimensional wave number, respectively. Furthermore, $W=w h / c_{0_{T}}$ denotes the nondimensional angular frequency and $A=\alpha h$. We depict the changes of $W$ and $C$ versus $K$ for the first three branches of dispersion relation (22) with the constant $A=0.01$ in Fig. 1 and Fig. 2, respectively. Moreover, we depict the effect of the heterogeneity on the change of $W$ versus $K$ for the first branch of dispersion relation (22) with the constants $A=0.01, A=0.03$, and $A=0.05$ in Fig. 3.

In this study, it is possible to choose the nonlinear material function as

$$
n_{T}=n_{0_{T}} \cosh ^{2}(\gamma y) \text {. }
$$




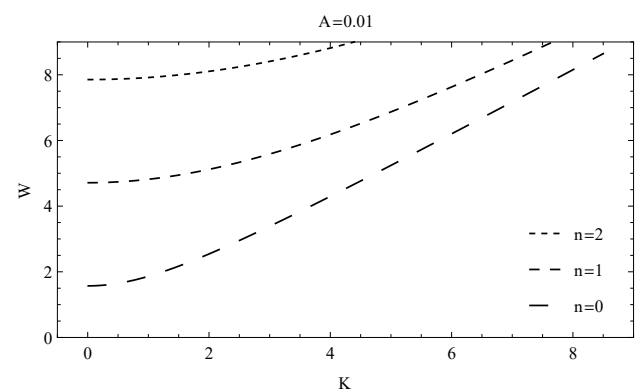

FiguRe 1. The change of $W$ versus $K$ for the first three branches of (22).

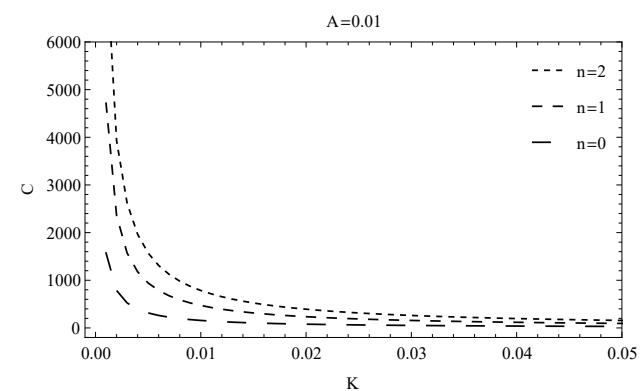

Figure 2. The change of $C$ versus $K$ for the first three branches of (22).

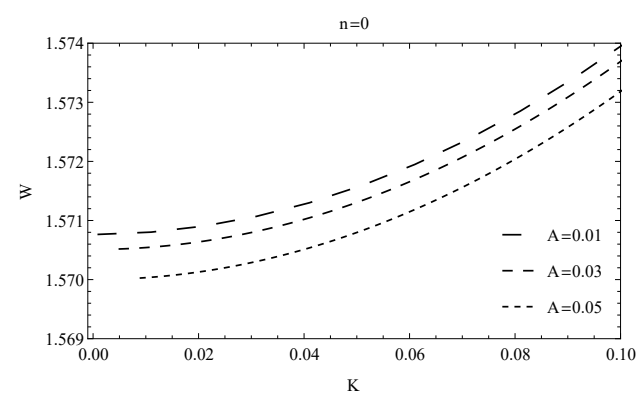

Figure 3. Heterogeneous effect on the change of $W$ versus $K$ for $n=0$.

Here, $n_{0_{T}}$ indicates a constant and $\gamma$ indicates a parameter. As a result of this study, we claim that if the layer consists of a hardening material, i.e. $n_{0_{T}}>0$, then $\Gamma \Delta<0$ for all phase velocities $c>c_{0_{T}}\left[1+\left(\alpha^{2} /\left(k^{2} l^{2}\right)\right)\right]^{1 / 2}$; but if the layer consists of a softening material, i.e. $n_{0_{T}}<0$, we refer to [14]. Hence, using the above review, we claim that if the layer consists of a hardening material, then the dark solitary SH waves will exist and propagate in such a layer.

We define a nondimensional constant as $\Lambda=\gamma h$ and depict the changes of $\Gamma,|\Delta|$, and $\Gamma|\Delta|$ versus $K$ for the first branch of dispersion relation (22) with the constants $A=0.01, \Lambda=0.04, n_{0_{T}}=1$ and $\mu_{0}=\rho_{0}=1$ in Fig. 4. Moreover, we depict the effects of the heterogeneities on the changes of $\Delta$ and $\Gamma \Delta$ versus $K$ for the first branch of dispersion relation (22) with the constants $A=0.01, A=0.03, A=0.05, \Lambda=0.04, n_{0_{T}}=1$ and $\mu_{0}=\rho_{0}=1$ in Fig. 5 and Fig. 6 , respectively.

The following specific hardening material is considered

$$
n_{0_{T}}=1, \mu_{0}=\rho_{0}=1, K=0.01, \varepsilon=0.01, \phi_{0}=0.01, B_{0}=0.01 .
$$


We show the effect of the heterogeneity on the deformation field of the plane $Z=0$ of the layer for dark solitary SH wave solution (30) considering the first branch of dispersion relation (22) with the constants $A=0.01, A=0.03$, and $A=0.05$ in Fig. 7 .

We depict the changes of $\Gamma,|\Delta|$, and $\Gamma|\Delta|$ versus $K$ for the first branch of dispersion relation (22) with the constants $A=0.01, \Lambda=0.5, n_{0_{T}}=1$ and $\mu_{0}=\rho_{0}=1$ in Fig. 8. We also depict the effects of the nonlinearities on the changes of $\Delta$ and $\Gamma \Delta$ versus $K$ for the first branch of dispersion relation (22) with the constants $A=0.01, \Lambda=0.5, \Lambda=0.7, \Lambda=$ $0.9, n_{0_{T}}=1$ and $\mu_{0}=\rho_{0}=1$ in Fig. 9 and Fig. 10, respectively.

Using specific hardening material (35), we show the effect of the nonlinearity on the deformation field of the plane $Z=0$ of the layer for dark solitary $\mathrm{SH}$ wave solution (30) considering the first branch of dispersion relation (22) with $\Lambda=0.5, \Lambda=0.7$, and $\Lambda=0.9$ in Fig. 11.

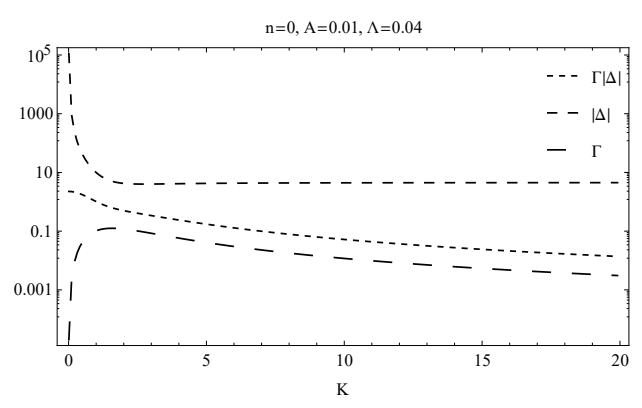

Figure 4. The changes of $\Gamma,|\Delta|$, and $\Gamma|\Delta|$ versus $K$ for $n=0$.

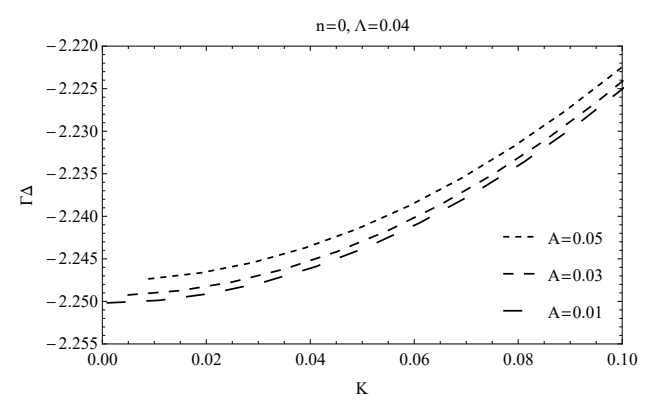

Figure 6. Heterogeneous effect on the change of $\Gamma \Delta$ versus $K$ for $n=0$.

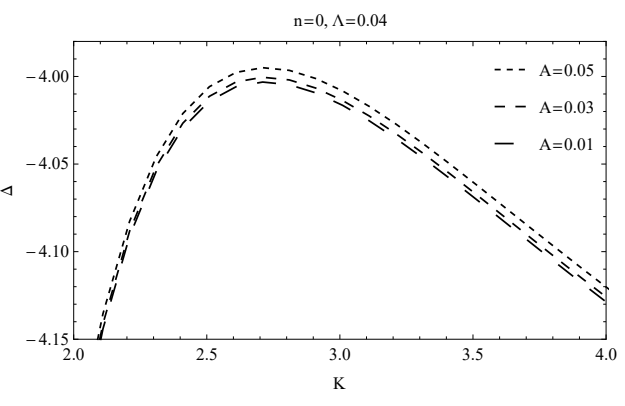

FiguRE 5. Heterogeneous effect on the change of $\Delta$ versus $K$ for $n=0$.

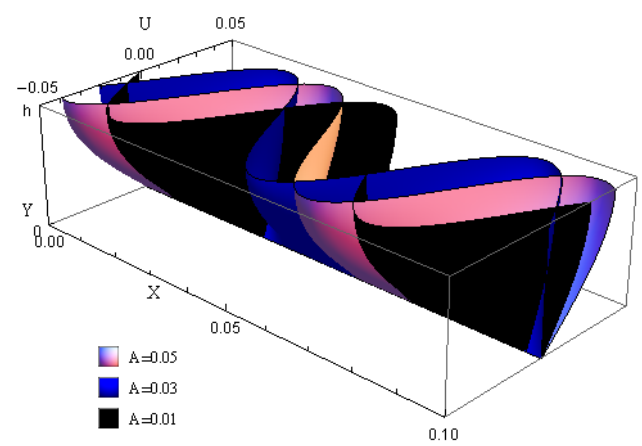

Figure 7. Heterogeneous effect on the deformation field of the plane $Z=0$ of the layer for (30). 
$\mathrm{n}=0, \mathrm{~A}=0.01, \Lambda=0.5$

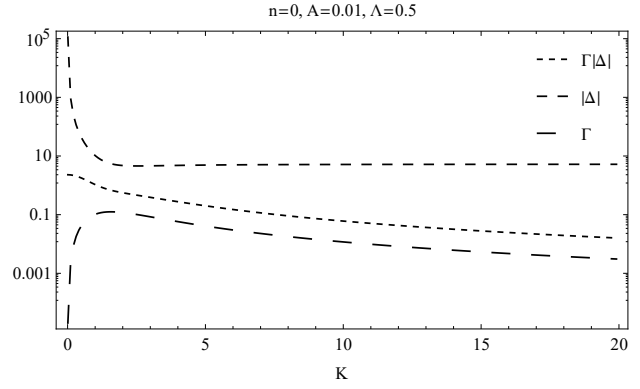

Figure 8. The changes of $\Gamma,|\Delta|$, and $\Gamma|\Delta|$ versus $K$ for $n=0$.

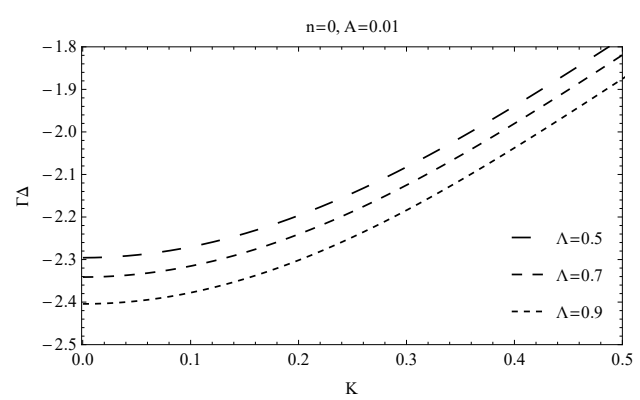

Figure 10. Nonlinea$r$ effect on the change of $\Gamma \Delta$ versus $K$ for $n=$ 0 .

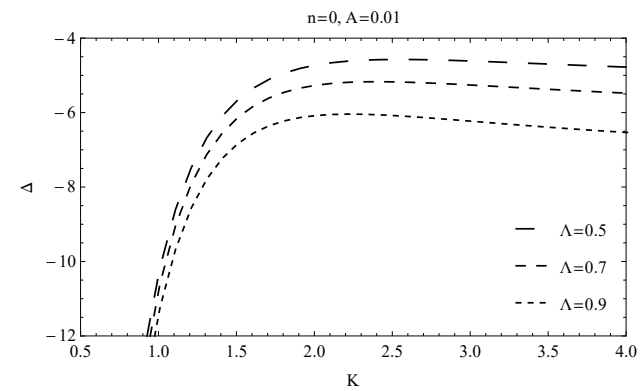

FiguRE 9. Nonlinear effect on the change of $\Delta$ versus $K$ for $n=0$.

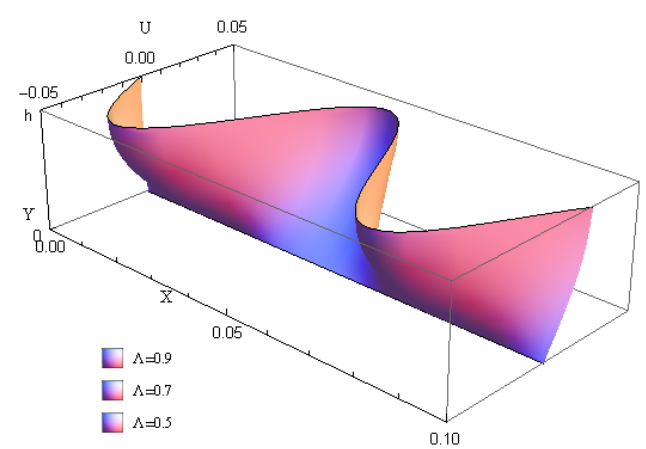

Figure 11. Nonlinear effect on the deformation field of the plane $Z=0$ of the layer for (30).

\section{REFERENCES}

[1] Teymur, M., (1988). Nonlinear modulation of Love waves in a compressible hyper-elastic layered half space, Int. J. Eng. Sci., 26, pp. 907-927.

[2] Mayer, A. P., (1995). Surface acoustic waves in nonlinear elastic media, Phys. Reports, 256, pp. 4-5.

[3] Fu, Y., (1994). On the propagation of nonlinear travelling waves in an incompressible elastic plate, Wave Motion, 19, pp. 271-292.

[4] Porubov, A. V. and Samsonov, A. M., (1995). Long nonlinear strain waves in layered elastic half space, Int. J. Nonlinear Mech., 30 (6), pp. 861-877.

[5] Pucci, E. and Saccomandi, G., (2013). Secondary motions associated with anti-plane shear in nonlinear isotropic elasticity, Q. Jl. Mech. Appl. Math., 66, pp. 221-239.

[6] Deliktas, E. and Teymur, M., (2018). Surface shear horizontal waves in a double-layered nonlinear elastic half space, IMA J. of Applied Math., 83 (3), pp. 471-495.

[7] Ahmetolan, S. and Teymur, M., (2003). Nonlinear modulation of SH waves in a two layered plate and formation of surface SH waves, Int. J. Nonlinear Mech., 38, pp. 1237-1250.

[8] Destrade, M., Goriely, M. A. and Saccomandi, G., (2011). Scalar evolution equations for shear waves in incompressible solids: a simple derivation of the Z, ZK, KZK and KP equations, Proc. R. Soc. A, 467, pp. 1823-1834.

[9] Teymur, M., Demirci, A. and Ahmetolan, S., (2014). Propagation of surface SH waves on a half space covered by a nonlinear thin layer, Int. J. Eng. Sci., 85, pp. 150-162.

[10] Demirkus, D. and Teymur, M., (2017). Shear horizontal waves in a nonlinear elastic layer overlying a rigid substratum, Hacettepe J. of Math. and Stats., 46 (5), pp. 801-815. 
[11] Kaplunov, J., Prikazchikov, D. A. and Prikazchikova, L. A., (2017). Dispersion of elastic waves in a strongly inhomogeneous three-layered plate, Int J. Solids Struc. , 113, pp. 169-179.

[12] Kaplunov, J. and Nobili, A., (2017). Multi-parametric analysis of strongly inhomogeneous periodic waveguides with internal cut-off frequencies, Math. Meth. App.Sci., 40 (9), pp. 3381-3392.

[13] Prikazchikova, L., Aydın, Y. E., Erbaș, B. and Kaplunov, J., (2018). Asymptotic analysis of an antiplane dynamic problem for a three-layered strongly inhomogeneous laminate, Math. and Mech. of Solids, https://doi.org/10.1177/1081286518790804.

[14] Demirkuş, D., (2018). Nonlinear bright solitary SH waves in a hyperbolically heterogenous layer, Int. J. Nonlinear Mech., 102, pp. 53-61.

[15] Demirkuş, D., (2019). Symmetric bright solitary SH waves in a nonlinear heterogeneous plate. Z. Angew. Math. Phy., 70 (2), no: 63.

[16] Demirkuş, D., (2019). Symmetric dark solitary SH waves in a nonlinear heterogeneous plate. Z. Angew. Math. Phy., 70 (4), no: 108.

[17] Demirkuş, D., (2018). Antisymmetric bright solitary SH waves in a nonlinear heterogeneous plate. Z. Angew. Math. Phy., 69 (5), no: 128.

[18] Jeffrey, A. and Kawahara, T., (1981). Asymptotic Methods in Nonlinear Wave Theory, Pitman, Boston.

[19] Peregrine, D. H., (1983). Water waves, non-linear Schrödinger equations and their solutions, J. Aust. Math. Soc. Ser. B., 25, pp. 16-43.

[20] Dodd, R. K., Eilbeck J. C., Gibbon, J. D., Morris, H. C., (1982). Solitons and Nonlinear Wave Equations, Academic Press, London.

[21] Ablowitz, M. J., Clarkson P. A., (1991). Solitons, Nonlinear Evolution Equations and Inverse Scattering, Cambridge University Press, Cambridge.

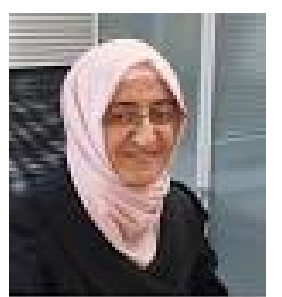

Dilek Demirkuş is an assistant professor at Beykent University and her research interests are wave propagation, nonlinear PDEs, asymptotic analysis, etc. She has published some research papers in some good journals. She is willing to work in the interdisciplinary area. 